Pacific Journal of Mathematic 


\section{SOME THEOREMS ON THE SCHUR DERIVATIVE}

\section{CARLITZ}

1. Introduction. Given the sequence $\left\{a_{m}\right\}$ and $p \neq 0$, Schur [5] defined the derivative $a_{m}^{\prime}$ by

$$
a_{m}^{\prime}=\Delta a_{m}=\left(a_{m+1}-a_{m}\right) / p^{m+1} ;
$$

higher derivatives are defined by means of

$$
a_{m}^{(r)}=\Delta^{r} a_{m}=\Delta\left(a_{m}^{(r-1)}\right), \quad a_{m}^{(0)}=a_{m} .
$$

In particular if $p$ is a prime, $a$ an integer and $a_{m}=a^{p^{m}}$, then by Fermat's theorem

$$
a_{m}^{\prime}=\left(a^{p^{m+1}}-a^{p^{m}}\right) / p^{m+1}
$$

is integral. Schur proved that if $p \nmid a$, then also the derivatives

$$
\Delta^{2} a^{p^{m}}, \Delta^{3} a^{p^{m}}, \cdots, \Delta^{p-1} a^{p^{m}}
$$

are all integral. Moreover if $a_{0}^{\prime} \equiv 0(\bmod p)$ then all the derivatives $\Delta^{r} a^{p^{m}}$ are integral, while if $a_{0}^{\prime} \not \equiv 0(\bmod p)$ then every number of $\Delta^{p} a^{p^{m}}$ has the denominator $p$.

A. Brauer [1] gave another proof of Schur's results. About the same time Zorn [6] proved these results by $p$-adic methods and indeed proved the following stronger theorem. For $x \equiv 1(\bmod p)$, define

$$
X_{m}=\left(x^{p^{m}}-1\right) / p^{m+1},
$$

and as above let $\Delta^{r} X_{m}$ denote the $r$-th derivative of $X_{m}$; then

$$
\Delta^{r} X_{m} \equiv \frac{(p-1)\left(p^{2}-1\right) \cdots\left(p^{r}-1\right)}{(r+1) !} X_{m}^{r+1}\left(\bmod p^{m}\right)
$$

provided $r<p$; for $r<p-2$, the congruence $(1.2)$ holds $\left(\bmod p^{m+1}\right)$. It is also shown that Schur's theorem is an easy consequence of Zorn's results. 
In the present paper we shall give a simple elementary proof of Zorn's congruences. In addition we prove, for example, that for $r \leq p$,

$$
\Delta^{r} a^{p^{m}} \equiv \frac{1}{r !} a^{p^{m}} q_{m}^{r} \frac{\prod_{i=1}^{r}\left(p^{i}-1\right)}{(p-1)^{r}}\left(\bmod p^{m}\right),
$$

where

$$
a^{(p-1)} p^{m}=1+p^{m+1} q_{m}
$$

for $r<p-1,(1.3)$ holds $\left(\bmod p^{m+1}\right)$.

We next $(\$ 4)$ extend Schur's and Zorn's theorems to algebraic numbers. In $\$ 5$ we consider a generalization of another kind suggested by the arithmetic function ( see for example [2, p. 84-86])

$$
F(a, m)=\sum_{d e=m} \mu(d) a^{e} .
$$

Finally $(\$ 6)$, we give some applications of Schur's theorem to the Euler and Bernoulli polynomials and numbers; the results are analogous to Kummer's congruences [3, Ch. 12]. In particular $\Delta^{r} E_{k+p^{m}}$ is integral $(\bmod p)$ for $p>2, r<p$, $r \leq m$; also $\Delta^{r}\left(B_{k+p^{m}} /\left(k+p^{m}\right)\right)$ is integral $(\bmod p)$ for $p-1 \nmid k+1, r<p$, $r \leq m$. Here $E_{k}$ and $B_{k}$ denote the Euler and Bernoulli numbers in the notation of Nörlund [3].

2. Formulas for $\Delta^{r} a_{m}$. We shall require some preliminary results.

Lemma 1. The following identity holds:

$$
\prod_{i=0}^{r=1}\left(x-p^{i}\right)=\sum_{i=0}^{r}(-1)^{i}\left[\begin{array}{l}
r \\
i
\end{array}\right] p^{i(i-1) / 2} x^{r-i}
$$

where

$$
\left[\begin{array}{c}
r \\
i
\end{array}\right]=\frac{\left(p^{r}-1\right)\left(p^{r-1}-1\right) \cdots\left(p^{r-i+1}-1\right)}{(p-1)\left(p^{2}-1\right) \cdots\left(p^{i}-1\right)}=\left[\begin{array}{c}
r \\
r-i
\end{array}\right],\left[\begin{array}{c}
r \\
0
\end{array}\right]=1 .
$$

Lemma 2. Put

$$
W_{k, r}=\sum_{i=0}^{k}(-1)^{i}\left[\begin{array}{c}
k \\
i
\end{array}\right]\left(\begin{array}{c}
p_{r}^{k-i} \\
r
\end{array}\right) p^{i(i-1) / 2},
$$


where $\left(\begin{array}{c}m \\ r\end{array}\right)$ denotes a binomial coefficient. Then

$$
w_{k, r}= \begin{cases}0 & (r<k) \\ \frac{1}{r !} \prod_{i=0}^{r-1}\left(p^{r}-p^{i}\right) & (r=k) \\ \frac{1}{r !} p^{k(k-1) / 2} U_{k, r} & (r>k),\end{cases}
$$

where $U_{k, r}$ is an integer.

Lemma 1 is will known. To prove Lemma 2, we note first that the binomial coefficient $\left(\begin{array}{l}x \\ r\end{array}\right)$ is a polynomial in $x$ of degree $r$. Since by $(2.1)$

$$
\sum_{i=0}^{k}(-1)^{i}\left[\begin{array}{c}
k \\
i
\end{array}\right] p^{i(i-1) / 2} p^{r(k-i)}=\prod_{i=0}^{k-1}\left(p^{r}-p^{i}\right),
$$

the several parts of ( 2.3$)$ follow without much difficulty.

LEMмA 3. For an arbitrary sequence $\left\{a_{m}\right\}$,

$$
\Delta^{r} a_{m}=p^{-r m-r(r+1) / 2} \sum_{i=0}^{r}(-1)^{i}\left[\begin{array}{l}
r \\
i
\end{array}\right] p^{i(i-1) / 2} a_{m+r-i}
$$

This formula, which is given by Schur, is easily proved. In view of (2.1) it can be put in the following symbolic form:

$$
\Delta^{r} a_{m}=p^{-r m-r(r+1) / 2} a^{m} \prod_{i=0}^{r-1}\left(a-p^{i}\right)
$$

where it is understood that after expansion of the right member $a^{k}$ is to be replaced by $a_{k}$.

Suppose now that $p \nmid a$ and put

$$
a^{(p-1) p^{m}}=1+p^{m+1} q_{m}
$$

so that $q_{m}$ is integral. Then by the binomial theorem we have

$$
a^{(p-1) p^{m+s}}=\sum_{i=0}^{p^{r}}\left(\begin{array}{c}
p^{s} \\
i
\end{array}\right) p^{(m+1) i} q_{m}^{i} \quad(r \geq s)
$$


and by $(2.4)$ this implies

$$
\begin{aligned}
& p^{r m+r(r+1) / 2} \Delta^{r} a^{(p-1) p^{m}} \\
& \left.=\sum_{s=0}^{r}(-1)^{r-s}\left[\begin{array}{c}
r \\
s
\end{array}\right] p^{(r-s)(r-s-1) / 2} \sum_{i=0}^{p^{r}}{ }_{i}^{p^{s}}\right) p^{(m+1) i} q_{m}^{i} \\
& =\sum_{i=0}^{p^{r}} p^{(m+1) i} q_{m}^{i} \sum_{s=0}^{r}(-1)^{r-s}\left[\begin{array}{l}
r \\
s
\end{array}\right]\left(\begin{array}{c}
p^{s} \\
i
\end{array}\right) p^{(r-s)(r-s-1) / 2} \\
& =\sum_{i=0}^{p^{r}} p^{(m+1) i} q_{m}^{i} W_{r, i} \\
& =\frac{1}{r !} p^{r m+r(r+1) / 2} q_{m}^{r} \prod_{i=1}^{r}\left(p^{i}-1\right) \\
& +\sum_{i=r+1}^{p^{r}} \frac{1}{i !} p^{(m+1) i+r(r-1) / 2} q_{m}^{i} U_{r, i}
\end{aligned}
$$

by (2.3); $W_{r, i}$ and $U_{r, i}$ have the same meaning as in Lemma 2 . We thus get

$$
\Delta^{r} a^{(p-1) p^{m}}=\frac{1}{r !} q_{m}^{r} \prod_{i=1}^{r}\left(p^{i}-1\right)+\sum_{i=r+1}^{p^{r}} \frac{1}{i !} p^{(m+1)(i-r)} q_{m}^{i} U_{r, i}
$$

i e next set up a similar formula for $\Delta^{r} q_{m}$, where $q_{m}$ is defined by $(2.6)$. Indeed substitution in $(2.4)$ gives

$$
\begin{aligned}
p^{r m+r(r+1) / 2} \Delta^{r} q_{m} & =\sum_{s=0}^{r}(-1)^{r-s}\left[\begin{array}{l}
r \\
s
\end{array}\right] p^{(r-s)(r-s-1) / 2-(m+s+1)}\left(a^{\left.(p-1) p^{m+s}-1\right)}\right. \\
& =\sum_{s=0}^{r}(-1)^{r-s}\left[\begin{array}{l}
r \\
s
\end{array}\right] p^{(r-s)(r-s-1) / 2-(m+s+1)} \sum_{i=1}^{p^{r}}\left(p_{i}^{s}\right) p^{(m+1) i} q_{m}^{i} \\
& =\sum_{i=1}^{p^{r}} p^{(m+1)(i-1)} q_{m}^{i} \sum_{s=0}^{r}(-1)^{r-s}\left[\begin{array}{c}
r \\
s
\end{array}\right]\left(\begin{array}{c}
p^{s} \\
i
\end{array}\right) p^{(r-s)(r-s-1) / 2-s} \\
& =\frac{1}{(r+1) !} p^{r m+r(r+1) / 2} q_{m}^{r+1} \prod_{i=1}^{r}\left(p^{i}-1\right) \\
& \quad \sum_{i=r+2}^{p^{r}} \frac{1}{i !} p^{(m+1)(i-1)+r(r-1) / 2} q_{m}^{i} U_{r, i}^{\prime},
\end{aligned}
$$


by a slight modification of Lemma 2 ; the coefficient $U_{r, i}^{\prime}$ is integral and is defined by

$$
\frac{1}{i !} p^{r(r-1) / 2} U_{r, i}^{\prime}=\sum_{s=0}^{r}(-1)^{s}\left[\begin{array}{l}
r \\
s
\end{array}\right]\left(p_{i}^{r-s}\right) p^{s(s-1) / 2-(r-s)} .
$$

Hence

$$
\Delta^{r} q_{m}=\frac{1}{(r+1) !} q_{m}^{r+1} \prod_{i=1}^{r}\left(p^{i}-1\right)+\sum_{i=r+2}^{p^{r}} \frac{1}{i !} p^{(m+1)(i-r-1)} q_{m}^{i} U_{r, i}^{\prime}
$$

Using the same method we can also evaluate $\Delta^{r} a^{p^{m}}$. It follows from $(2.6)$ that

$$
a^{p^{m+s}}=a^{p^{m}}\left(1+p^{m+1} q_{m}\right)^{e_{s}} \quad\left(e_{s}=\frac{p^{s}-1}{p-1}\right)
$$

and thus substitution in $(2.4)$ yields

$$
\begin{aligned}
p^{r m+r(r+1) / 2} \Delta^{r} a^{p^{m}} & =a^{p^{m}} \sum_{s=0}^{r}(-1)^{r-s}\left[\begin{array}{l}
r \\
s
\end{array}\right] p^{(r-s)(r-s-1) / 2} \sum_{i=0}^{e_{r}}\left(\begin{array}{c}
e_{s} \\
i
\end{array}\right) p^{(m+1) i} q_{m}^{i} \\
& =a^{p^{m}} \sum_{i=0}^{e_{r}} p^{(m+1) i} q_{m}^{i} \sum_{s=0}^{r}(-1)^{r-s}\left[\begin{array}{c}
r \\
s
\end{array}\right]\left(\begin{array}{c}
e_{s} \\
i
\end{array}\right) p^{(r-s)(r-s-1) / 2} .
\end{aligned}
$$

Since $\left(\begin{array}{c}e_{s} \\ i\end{array}\right)$ is a polynomial in $p^{s}$ of degree $i$, the same reasoning as before applies and we get after a little manipulation

$$
\begin{aligned}
& \Delta^{r} a^{p^{m}}=\frac{1}{r !} a^{p^{m}} q_{m}^{r} \frac{\prod_{i=1}^{r}\left(p^{i}-1\right)}{(p-1)^{r}} \\
& \quad+a^{p^{m}} \sum_{i=r+1}^{e_{r}} \frac{1}{i !} p^{(m+1)(i-r)} q_{m}^{i} U_{r, i}^{\prime \prime}
\end{aligned}
$$

where $U_{r, i}^{\prime \prime}$ is integral.

Comparison of (2.7) and (2.10) shows that (2.7) is included in (2.10). Indeed it is easy to set up the following formula which includes both (2.7) and $(2.10)$ : 


$$
\begin{aligned}
\Delta^{r} a^{k p^{m}}=\frac{1}{r !} a^{k p^{m}} q_{m}^{r} k^{r} \frac{\prod_{i=1}^{r}\left(p^{i}-1\right)}{(p-1)^{r}} \\
\\
+a^{k p^{m}} \sum_{i=r+1}^{e_{r}} \frac{1}{i !} p^{(m+1)(i-r)} q_{m}^{i} V_{r, i},
\end{aligned}
$$

where $V_{r, i}=V_{r, i}^{(k)}$ is integral and $k \geq 1$. The proof of (2.11) is exactly like the proof of (2.10); the first step is to raise both members of $(2.9)$ to the $k$-th power.

3. The main results. In order to make use of (2.7) and (2.10) it is evidently necessary to examine $p^{(m+1)(i-r)} / i$ ! . We suppose $i>r, r \leq p$. Then in the first place it is easily seen [6, p. 462] that $p^{i-r} / i !$ is integral $(\bmod p)$, and a simple discussion shows that $p^{i-r} / i$ ! is divisible by $p$ unless $(\mathrm{i}) i=p, r=p-1$, or (ii) $i=p+1, r=p$. We now state:

THEOREM 1. The derivative $\Delta^{r} a^{(p-1) p^{m}}$ is integral for $1 \leq r \leq p-1$, while $\Delta^{p} a^{(p-1) p^{m}}$ has the denominator $p$ provided $a^{p-1} \equiv 1\left(\bmod p^{2}\right) ;$ if $a^{p-1} \equiv 1$ $\left(\bmod p^{2}\right)$ then all $\Delta^{r} a^{(p-1) p^{m}}$ are integral.

THEOREM 2. For $1 \leq r \leq p, m \geq 0$,

$$
\Delta^{r} a^{(p-1) p^{m}} \equiv \frac{1}{r !} q_{m}^{r} \prod_{i=1}^{r}\left(p^{i}-1\right) \quad\left(\bmod p^{m}\right)
$$

if $r<p-1$, the congruence is valid $\left(\bmod p^{m+1}\right)$.

THEOREM 3. The derivative $\Delta^{r} a^{p^{m}}$ is integral for $1 \leq r \leq p-1$, while $\Delta^{p} a^{p^{m}}$ has the denominator $p$ provided $a^{p-1} \equiv 1\left(\bmod p^{2}\right) ;$ if $a^{p-1} \equiv 1\left(\bmod p^{2}\right)$ then all $\Delta^{r} a^{(p-1) p^{m}}$ are integral.

THEOREM 4. For $1 \leq r \leq p, m \geq 0$,

$$
\Delta^{r} a^{p^{m}} \equiv \frac{1}{r !} a^{p^{m}} q_{m}^{r} \frac{\prod_{i=1}^{r}\left(p^{i}-1\right)}{(p-1)^{r}}\left(\bmod p^{m}\right)
$$

if $r<p-1$, the congruence is valid $\left(\bmod p^{m+1}\right)$.

If we make use of (2.11) rather than (2.7) or (2.10) we get the following more general result.

THEOREM $4^{\prime}$. For $1 \leq r \leq p, m \geq 0$ 


$$
\Delta^{r} a^{k p^{m}} \equiv \frac{1}{r !} a^{k p^{m}} q_{m}^{r} k^{r} \frac{\prod_{i=1}^{r}\left(p^{i}-1\right)}{(p-1)^{r}}\left(\bmod p^{m}\right)
$$

if $r<p-1$, the congruence is valid $\left(\bmod p^{m+1}\right)$.

To apply (2.8) we first examine $p^{i-r-1} / i$ ! for $i>r+1, r+1 \leq p$. We have:

THE OR EM 5. The derivative $\Delta^{r} q_{m}$ is integral for $1 \leq r \leq p-2$, while $\Delta^{p-1} q_{m}$ has the denominator $p$ provided $a^{p-1} \equiv 1\left(\bmod p^{2}\right) ;$ if $a^{p-1} \equiv 1\left(\bmod p^{2}\right)$ then all $\Delta^{r} q_{m}$ are integral.

THEOREM 6. For $1 \leq r \leq p-1, m \geq 0$,

$$
\Delta^{r} q_{m} \equiv \frac{1}{(r+1) !} q_{m}^{r+1} \prod_{i=1}^{r}\left(p^{i}-1\right) \quad\left(\bmod p^{m}\right) ;
$$

if $r<p-2$, the congruence is valid $\left(\bmod p^{m+1}\right)$.

Theorem 3 is of course Schur's theorem; Theorems 5 and 6 are due to Zorn. The remaining theorems are presumably new.

4. Generalization for algebraic numbers. Let $k$ be an algebraic number field of degree $n$ and let $\{$ denote a prime ideal of $k$; also let

$$
N f=p^{f} ; \quad h^{e} \mid p, \quad p^{e+1}+p ;
$$

for simplicity we assume $p>n$. If $\alpha k$ is integral $(\bmod p)$ and $p \nmid \alpha$, then by Fermat's Theorem

$$
\alpha^{p^{f-1}}=1+\beta, \quad \beta \equiv 0 \quad(\bmod \wp) .
$$

It follows from $(4.2)$ that

$$
\alpha^{\left(p^{f}-1\right) p^{m}}=1+\beta_{m}, \quad \beta_{m} \equiv 0 \quad\left(\bmod p^{m e+1}\right),
$$

while (4.3) implies

$$
a^{\left(p^{f}-1\right) p^{m+s}}=\sum_{i=0}^{p^{r}}\left(\begin{array}{c}
p^{s} \\
i
\end{array}\right) \beta_{m}^{i} \quad(r \geq s) .
$$

Then, exactly as in $\$ 2$, 


$$
\begin{aligned}
p^{r m+r(r+1) / 2} \Delta^{r} a^{\left(p^{f}-1\right) p^{m}} & =\sum_{s=0}^{r}(-1)^{r-s}\left[\begin{array}{l}
r \\
s
\end{array}\right] p^{(r-s)(r-s-1) / 2} \sum_{i=0}^{p^{r}}\left(P_{i}^{s}\right) \beta_{m}^{i} \\
& =\sum_{i=0}^{p^{r}} \beta_{m}^{i} \sum_{s=0}^{r}(-1)^{r-s}\left[\begin{array}{l}
r \\
s
\end{array}\right]\left(\begin{array}{c}
p_{i}^{s} \\
i
\end{array}\right) p^{(r-s)(r-s-1) / 2}
\end{aligned}
$$

application of Lemma 2 now leads to

(4.5) $\Delta^{r} \alpha^{\left(p^{f-1) p^{m}}\right.}=\frac{1}{r !} p^{-r(m+1)} \beta_{m}^{r} \prod_{i=1}^{r}\left(p^{i}-1\right)+\sum_{i=r+1}^{p^{r}} \frac{1}{i !} p^{-r(m+1)} \beta_{m^{\prime}}^{i} \omega_{r, i}$, where $\omega_{r, i}$ is integral. Note that for $e>1$ the right member of (4.5) need not be integral. Accordingly we assume $e=1$; the assumption $p>n$ is then no longer needed.

We now have:

THEOREM 7. Let $N p=p^{f}, p^{2} \nmid p$, $\nmid \nmid a$; then $\Delta^{r} a^{\left(p^{f}-1\right) p^{m}}$ is integral for $1 \leq r \leq p-1$, while $\Delta^{p} a^{\left(p^{f-1}\right) p^{m}}$ has the denominator $p$ provided $a^{p^{f-1}} \equiv 1$ $\left(\bmod \hat{p}^{2}\right) ;$ if $\alpha^{p^{f-1}} \equiv 1\left(\bmod \mathfrak{p}^{2}\right)$ then all $\Delta^{r} \alpha^{\left(p^{f-1}\right) p^{m}}$ are integral.

THEOREM 8. With the hypotheses of Theorem 7,

$$
\Delta^{r} \alpha^{\left(p^{f}-1\right) p^{m}} \equiv \frac{1}{r !}\left(\frac{\beta_{m}}{p^{m+1}}\right)^{r} \prod_{i=1}^{r}\left(p^{i}-1\right) \quad\left(\bmod p^{m}\right)
$$

for $r \leq p$; if $r<p-1$ the congruence is valid $\left(\bmod p^{m+1}\right)$.

In order to extend Theorems 3 and $4^{\prime}$ it is convenient to suppose that $f$ is a prime ideal of the first degree. The following two theorems may be proved.

THEOREM 9. Let $N p=p, \wp^{2} \nmid p, \wp \nmid \alpha$; then $\Delta^{r} \alpha^{p^{m}}$ is integral for $1 \leq r \leq$ $p-1$, while $\Delta^{p} a^{p^{m}}$ has the denominator $p$ provided $a^{p-1} \equiv 1\left(\bmod p^{2}\right) ;$ if $a^{p-1} \equiv$ $1\left(\bmod \mathfrak{p}^{2}\right)$ then all $\Delta^{r} \alpha^{p^{m}}$ are integral.

THEOREM 10. With the hypotheses of Theorem 9,

$$
\Delta^{r} \alpha^{k p^{m}} \equiv \frac{1}{r !}\left(\frac{k \beta_{m}}{p^{m+1}}\right)^{r} \frac{\prod_{i=1}^{r}\left(p^{i}-1\right)}{(p-1)^{r}} \quad\left(\bmod p^{m}\right)
$$

for $r \leq p$; if $r<p-1$ the congruence is valid $\left(\bmod p^{m+1}\right)$. 
For brevity we omit the extension of Theorems 5 and 6 for algebraic numbers.

5. Another generalization. Changing slightly the notation (1.1) we put

$$
\Delta_{p} a_{m p i}=\left(a_{m p} i+1-a_{m p^{i}}\right) / p^{i+1},
$$

and

$$
\Delta_{p}^{r} a_{m p} i=\left(\Delta_{p}^{r-1} a_{m p} i+1-\Delta_{p}^{r-1} a_{m p} i\right) / p^{i+1} .
$$

Then clearly $\Delta_{p} \Delta_{q}=\Delta_{q} \Delta_{p}$. If $a$ and $k$ are arbitrary integers then if follows from a well-known theorem concerning (1.4) that

$$
\delta_{k} a^{k}=\Delta_{p_{1}} \cdots \Delta_{p_{s}} a^{k} \quad\left(k=p_{1}^{e_{1}} \cdots p_{s}^{e_{s}}\right)
$$

is integral. In view of Schur's theorem we can state the following generalization.

Тнеовем 11. Let $(a, k)=1$ and let $r<$ the smallest prime dividing $k$; define

$$
\delta_{k}^{r} a^{k}=\delta_{k} \delta_{k}^{r-1} a^{k}
$$

Then $\delta_{k}^{r} a_{k}$ is integral for $k>1$.

Indeed because of the commutativity of the operators $\Delta_{p_{i}}$ we need only observe that (5.2) and (5.3) imply

$$
\delta_{k}^{r} a^{k}=\Delta_{p_{1}}^{r} \cdots \Delta_{p_{s}}^{r} a^{k}
$$

and the theorem follows immediately.

The restriction $(a, k)=1$ can be removed by taking $k$ sufficiently large as we shall see below.

A slight extension of Theorem 11 is contained in:

THEOREM 12. Let

$$
(a, k)=1, \quad k=p_{1}^{e_{1}} \cdots p_{s}^{e_{s}},
$$

and let $r_{i}<p_{i}, j=1, \cdots, s ;$ then

$$
\Delta_{p_{1}}^{r_{1}} \cdots \Delta_{p_{s}}^{r_{s}} a^{k}
$$

is integral for all $k>1$.

We remark that the function defined in (5.2) can also be expressed in the form 


$$
\delta_{k} a^{k}=\frac{(-1)^{s}}{k_{1}} \sum_{d \mid k} \mu(d) a^{d k},
$$

where $\mu(d)$ is the Möbius function and

$$
k_{1}=p_{1}^{e_{1}+1} \cdots p_{s}^{e_{s}+1}
$$

similarly (5.3) becomes

$$
\delta_{k}^{r} a^{k}=\frac{(-1)^{s}}{k_{1}} \sum_{d \mid k} \mu(d) \delta_{k}^{r-1} a^{d k} .
$$

Formulas of a different kind can be obtained by applying (2.4) to (5.4) and (5.5); for example, (2.5) suggests the following symbolic formula:

$$
\delta_{k}^{r} a^{k}=k^{-r} \prod_{j=1}^{s} p_{j}^{r(r+1) / 2} \cdot \prod_{j=1}^{s} a_{j}^{e_{j}} \prod_{i=0}^{r=1}\left(a_{j}-p_{j}^{i}\right),
$$

where after expansion $a_{1}^{f_{1}} \ldots a_{s}^{f_{s}}$ is to be replaced by $a^{m}$,

$$
m=p_{1}^{f_{1}} \ldots p_{s}^{f_{s}} \text {. }
$$

A similar but slightly more complicated formula can be stated for (5.5). We shall omit the generalization of Theorems 11 and 12 to algebraic numbers.

6. Applications. In the theorems of $\S 2$ it is assumed that $p \nmid a$. However Theorem 3, for example, is easily extended to the case $p \mid a$. We can state that $\Delta^{r} a^{p^{m}}$ is integral for $r \leq p-1$ and arbitrary $a$ provided $m \geq r$. For let $p \mid a$; then, in view of (2.4), it is only necessary to verify that

$$
p^{m+r-i}+\frac{1}{2} i(i-1) \geq r m+\frac{1}{2} r(r+1)
$$

for $0 \leq i \leq r \leq p-1, r \geq m$. This can be proved by induction with respect to $m$. In the next place since Theorem 11 is a direct consequence of Theorem 3 we infer that it also holds for all $a$ provided $r \leq \min \left(e_{1}, \ldots, e_{s}\right)$ in the notation of Theorem 11.

Now consider the number

$$
C_{k}=\sum_{a=1}^{n} A_{a} a^{k},
$$


where $A_{a}$ denote integers $(\bmod p)$ and $n \geq 1$ is arbitrary. Then

$$
\Delta^{r} C_{k+p} m=\sum_{a=1}^{n} A_{a} \Delta^{r} a^{k+p^{m}}
$$

so that by the remark in the previous paragraph $\Delta^{r} C_{p^{m}}$ is certainly integral $(\bmod p)$ provided $r \leq p-1$ and $r \leq m$. In the second place we may apply the operator $\delta_{k}^{r}$ defined in $(5.2)$ and $(5.3)$ and get

$$
\delta_{k}^{r} C_{h+k}=\sum_{a=1}^{n} A_{a} \delta_{k}^{r} a^{h+k}
$$

we infer that $\delta_{k}^{r} C_{k}$ is integral provided $r<$ the smallest prime dividing $k$ and $r \leq \min \left(i_{1}, \cdots, i_{s}\right)$, the notation being that of (5.2). Indeed a somewhat more general result can be obtained by applying Theorem 15, namely,

$$
\Delta_{p_{1}}^{r_{1}} \cdots \Delta_{p_{s}}^{r_{s}} C_{h+k}
$$

is integral provided $r_{t}<p_{t}, r_{t} \leq e_{t}, t=1, \cdots, s$.

As an instance of (6.1) we take the well-known formula for the Euler polynomial

$$
E_{m}(x)=\sum_{s=0}^{m} \frac{1}{2^{s}} \sum_{i=0}^{s}(-1)^{i}\left(\begin{array}{c}
s \\
i
\end{array}\right)(x+i)^{m}
$$

(We use the notation of Nörlund [4] for the Euler and Bernoulli polynomials.) If $p>2$ and $x$ is integral $(\bmod p)$ the preceding discussion applies. In particular using (2.4) we have:

THEOREM 13. Let $p>2$ and $x$ be integral $(\bmod p)$. Then

$$
\Delta^{r} E_{k+p} m(x)=p^{-r m-r(r+1) / 2} \sum_{i=0}^{r}(-1)^{i}\left[\begin{array}{l}
r \\
i
\end{array}\right] p^{i(i-1) / 2} E_{k+p^{m-i}}(x)
$$

is integral $(\bmod p)$ provided $r<p, r \leq m$.

For brevity we omit the generalizations corresponding to (6.3) and (6.4). The special case

$$
\sum_{d e=m} \mu(d) E_{k+e}(x) \equiv 0 \quad(\bmod m)
$$


may be noted

As for the Bernoulli polynomials, it can be shown that if $p \nmid a$ and $x$ is integral $(\bmod p)$ then a formula of the type $(6.1)$ holds for

$$
\beta_{k}(x)=\frac{a^{k+1}-1}{k+1} B_{k+1}(x) .
$$

(See for example Nielsen [3, Ch.14].) Thus it follows that

$$
\Delta^{r} \beta_{k+p^{m}}(x)=p^{-r m-r(r+1) / 2} \sum_{i=0}^{r}(-1)^{i}\left[\begin{array}{c}
r \\
i
\end{array}\right] p^{i(i-1) / 2} \beta_{k+p^{m-i}}(x)
$$

is integral for $r<p, r \leq m$. If now we assume $p-1 \nmid k$ and take $a$ a primitive root $(\bmod p)$ such that $a^{p-1} \equiv 1\left(\bmod p^{r}\right)$ we get:

THEOREM 14. Let $p>2$ and $x$ be integral $(\bmod p) ;$ put $H_{k}(x)=B_{k}(x) / k$. Then if $p-1 \nmid k+1$,

$$
\Delta^{r} H_{k+p^{m}}(x)=p^{-r m-r(r+1) / 2} \sum_{i=0}^{r}(-1)^{i}\left[{ }_{i}^{r}\right] p^{i(i-1) / 2} H_{k+p^{m-i}}(x)
$$

is integral for $r<p, r \leq m$.

Finally corresponding to (6.6) we state

$$
\sum_{d e=m} \mu(d) \beta_{k+e}(x) \equiv 0 \quad(\bmod m)
$$

for $\beta_{k}(x)$ as defined in (6.7).

\section{REFERENCES}

1. A. Brauer, Über eine Erweiterung des kleinen Fermatschen Satzes, Math. Z. 42 (1937), 255-262.

2. L. E. Dickson, History of the theory of numbers, vol. 1, Washington, 1919.

3. Niels Nielsen, Traité élémentaire des nombres de Bernoulli, Paris, 1923.

4. N. E. Nörlund, Differenzenrechnung, Berlin, 1924.

5. I. Schur, Ein Beitrag zur elementaren Zahlentheorie, Sitzungsberichte der Preussischen Akademie der Wissenschaften (1933), 145-151. 451-464.

6. Max Zorn, p-adic analysis and elementary number theory, Ann. of Math. (2) 38(1937) 


\section{PACIFIC JOURNAL OF MATHEMATICS}

\section{EDITORS}

\section{R. U. FOEINSON}

University of California

Berkeley 4, California

E. HewitT

University of Washington

Seattle 5 , Washington
P. P. DILWOR TH

California Institute of Technology

Pasadena 4, California

E. F. BECKENBACH

University of California

Los Angeles 24, California

\section{ASSOCIATE EDITORS}

$\begin{array}{llll}\text { H. BUSEMANN } & \text { P. R. HALMOS } & \text { BØRGE JESSEN } & \text { J. J. STOKER } \\ \text { HERBERT FFDERER } & \text { IIEINZ HOPF } & \text { PAUL LÉVY } & \text { E. G. STRAUS } \\ \text { MARSHALL, IIALI } & \text { R. D. JAMES } & \text { GEORGE PÓLYA } & \text { KÖSAKU YOSIDA }\end{array}$

\section{SPONSORS}

UNIVERSITY OF BRITISH COLUMBIA

CALIFORNIA INSTITUTE OF TECHNOLOGY.

UNIVERSITY OF CAIJIFORNIA, BERKELEY

UNIVERSITY OF CAIJIFORNIA, DAVIS

UNIVERSITY OF CALIFORNIA, LOS ANGELES

UNIVERSITY OF CALIFORNI A, SANTA BARBARA

UNIVERSITY OF NEVADA

OREGON STATE COLLEGE

UNIVERSITY OF OREGON

\author{
UNIVERSITY OF SOU TIERN CALIFORNIA \\ STANFORD RESEARCH INSTITUTE \\ STANFORD UNIVERSITY \\ WASHINGTON STATE COLLEGE \\ UNIVERSITY OF WASHINGTON \\ AMERICAN MATHEMATICAL SOCIETY \\ NATIONAL BUREAU OF STANDARDS, \\ INSTITUTE FOR NUMERICAL ANALYSIS
}

Mathematical papers intended for publication in the Pacific Journal of Mathematics should be typewritten (double spaced), and the author should keep a complete copy. Manuscripts may be sent to any of the editors except Robinson, whose term expires with the completion of the present volume; they might also be sent to M.M. Schiffer, Stanford University, Stanford, California, who is succeeding Robinson. All other communications to the editors should be addressed to the managing editor, E. F. Beckenbach, at the address given above.

Authors are entitled to receive 100 free reprints of their published papers and may obtain additional copies at cost.

The Pacific Journal of Mathematics is published quarterly, in March, June, September, and December. The price per volume (4 numbers) is $\$ 8.00$; single issues, $\$ 2.50$. Special price to individual faculty members of supporting institutions and to individual members of the American Mathematical Society: $\$ 4.00$ per volume; single issues, $\$ 1.25$.

Subscriptions, orders for back numbers, and changes of address should be sent to the publishers, University of California Press, Berkeley 4, California.

Printed at Ann Arbor, Michigan. Entered as second class matter at the Post Office, Berkeley, California.

\section{UNIVERSITY OF CALIFORNIA PRESS • BERKELEY AND LOS ANGELES}




\section{Pacific Journal of Mathematics}

\section{Vol. 3, No. 2 \\ April, 1953}

William George Bade, An operational calculus for operators with spectrum

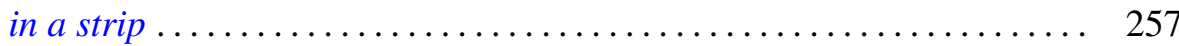

E. F. Beckenbach and Lloyd Kenneth Jackson, Subfunctions of several variables ..................................... 291

David Blackwell, Extension of a renewal theorem ................. 315

L. Carlitz, Some theorems on the Schur derivative ................ 321

Paul Arnold Clement, Generalized convexity and surfaces of negative curvature..................................... 333

Merrill M. Flood, On the Hitchcock distribution problem ............... 369

Watson Bryan Fulks, On the unique determination of solutions of the heat

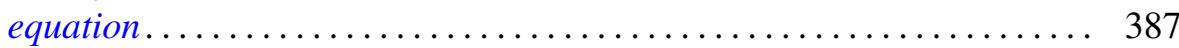

John W. Green, Length and area of a convex curve under affine transformation .................................... 393

William Gustin, An isoperimetric minimax .................. 403

Arthur Eugene Livingston, Some Hausdorff means which exhibit the Gibbs'

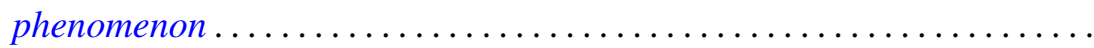

Charles Loewner, On generation of solutions of the biharmonic equation in the plane by conformal mappings ..................... 417

Gábor Szegő, Remark on the preceding paper of Charles Loewner ....... 437

Imanuel Marx and G. Piranian, Lipschitz functions of continuous

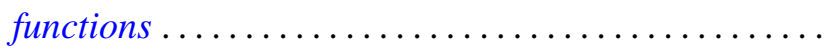

Ting-Kwan Pan, The spherical curvature of a hypersurface in Euclidean space ..

Ruth Lind Potter, On self-adjoint differential equations of second order ...

E. H. Rothe, A note on the Banach spaces of Calkin and Morrey...

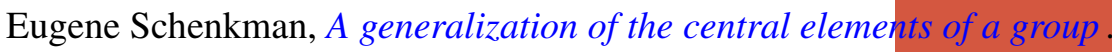

A. Seidenberg, A note on the dimension theory of rings .. . . 